\title{
On the Caffarelli-Kohn-Nirenberg Inequalities: Sharp Constants, Existence (and Nonexistence), and Symmetry of Extremal Functions
}

\author{
FLORIN CATRINA
}

AND

ZHI-QIANG WANG

Utah State University

Dedicated to Professor L. Nirenberg on the occasion of his $75^{\text {th }}$ birthday

\begin{abstract}
Consider the following inequalities due to Caffarelli, Kohn, and Nirenberg [6]:

$$
\left(\int_{\mathbb{R}^{N}}|x|^{-b p}|u|^{p} d x\right)^{2 / p} \leq C_{a, b} \int_{\mathbb{R}^{N}}|x|^{-2 a}|\nabla u|^{2} d x
$$

where, for $N \geq 3,-\infty<a<(N-2) / 2, a \leq b \leq a+1$, and $p=$ $2 N /(N-2+2(b-a))$. We shall answer some fundamental questions concerning these inequalities such as the best embedding constants, the existence and nonexistence of extremal functions, and their qualitative properties. While the case $a \geq 0$ has been studied extensively and a complete solution is known, little has been known for the case $a<0$. Our results for the case $a<0$ reveal some new phenomena which are in striking contrast with those for the case $a \geq 0$. Results for $N=1$ and $N=2$ are also given. (C) 2001 John Wiley \& Sons, Inc.
\end{abstract}

\section{Introduction}

In [6], among a much more general family of inequalities, Caffarelli, Kohn, and Nirenberg established the following inequalities: For all $u \in C_{0}^{\infty}\left(\mathbb{R}^{N}\right)$,

$$
\left(\int_{\mathbb{R}^{N}}|x|^{-b p}|u|^{p} d x\right)^{2 / p} \leq C_{a, b} \int_{\mathbb{R}^{N}}|x|^{-2 a}|\nabla u|^{2} d x
$$

where, for $N \geq 3$,

(1.2) $-\infty<a<\frac{N-2}{2}, \quad a \leq b \leq a+1, \quad$ and $\quad p=\frac{2 N}{N-2+2(b-a)}$. 
The cases $N=2$ and $N=1$ will be treated in a separate section. The conditions for these cases are, for $N=2$,

$$
-\infty<a<0, \quad a<b \leq a+1, \quad \text { and } \quad p=\frac{2}{b-a}
$$

and, for $N=1$,

(1.4) $-\infty<a<-\frac{1}{2}, \quad a+\frac{1}{2}<b \leq a+1, \quad$ and $\quad p=\frac{2}{-1+2(b-a)}$.

Let $D_{a}^{1,2}\left(\mathbb{R}^{N}\right)$ be the completion of $C_{0}^{\infty}\left(\mathbb{R}^{N}\right)$ with respect to the inner product

$$
(u, v)=\int_{\mathbb{R}^{N}}|x|^{-2 a} \nabla u \cdot \nabla v d x .
$$

Then we see that (1.1) holds for $u \in \mathscr{D}_{a}^{1,2}\left(\mathbb{R}^{N}\right)$. We define

$$
S(a, b)=\inf _{u \in \mathcal{D}_{a}^{1,2}\left(\mathbb{R}^{N}\right) \backslash\{0\}} E_{a, b}(u),
$$

to be the best embedding constants, where

$$
E_{a, b}(u)=\frac{\int_{\mathbb{R}^{N}}|x|^{-2 a}|\nabla u|^{2} d x}{\left(\int_{\mathbb{R}^{N}}|x|^{-b p}|u|^{p} d x\right)^{2 / p}} .
$$

The extremal functions for $S(a, b)$ are ground state solutions of the Euler equation

$$
-\operatorname{div}\left(|x|^{-2 a} \nabla u\right)=|x|^{-b p} u^{p-1}, \quad u \geq 0, \quad \text { in } \mathbb{R}^{N} .
$$

This equation is regarded as a prototype of more general nonlinear degenerate elliptic equations from physical phenomena (e.g., $[2,12]$ and references therein).

Note that the Caffarelli-Kohn-Nirenberg inequalities (1.1) (see also generalizations in [19] by Lin) contain the classical Sobolev inequality ( $a=b=0$ ) and the Hardy inequality $(a=0, b=1)$ as special cases, which have played important roles in many applications by virtue of the complete knowledge about the best constants, extremal functions, and their qualitative properties (see e.g., $[6,13,15,18]$ and references therein). Thus it is a fundamental task to study the best constants, existence (and nonexistence) of extremal functions, as well as their qualitative properties in inequality (1.1) for parameters $a$ and $b$ in the full parameter domain (1.2).

Much progress has been made for the parameter region

$$
0 \leq a<\frac{N-2}{2}, \quad a \leq b \leq a+1,
$$

(to which we shall refer as the " $a$-nonnegative region"). In [1, 23], the best constant and the minimizers for the Sobolev inequality $(a=b=0)$ were given by Aubin and Talenti. In [18], Lieb considered the case $a=0,0<b<1$, and gave the best constants and explicit minimizers. In [11], Chou and Chu considered the full $a$-nonnegative region and gave the best constants and explicit minimizers. Also for this $a$-nonnegative region, Lions in [22] (for $a=0$ ) and Wang and Willem 
(for $a>0$ ) in [25] established the compactness of all minimizing sequences up to dilations provided $a \leq b<a+1$. The symmetry of the minimizers has also been studied in [11] and [18]. In fact, all nonnegative solutions in $\mathscr{D}_{a}^{1,2}\left(\mathbb{R}^{N}\right)$ for the corresponding Euler equation (1.8) are radially symmetric (in the case $a=b=0$, they are radial with respect to some point) and explicitly given (see $[1,11,18,23])$. This was established in [11], where a generalization of the moving plane method was used (e.g., [5, 10, 14]).

On the other hand, it seems that little is known for parameters in the $a$-negative region

$$
-\infty<a<0, \quad a \leq b \leq a+1 .
$$

This also applies to $N=1$ and $N=2$, with $b$ in the corresponding intervals (1.4) and (1.3). The case $-1<a<0$ and $b=0$ was treated recently by Caldiroli and Musina in [7], who gave the existence of ground states. The goal of this paper is to settle some of the fundamental questions concerning inequalities (1.1) with parameters in the $a$-negative region, such as the best constants, the existence and nonexistence of minimizers, and the symmetry properties of minimizers. For the $a$-negative region we shall reveal new phenomena that are strikingly different from those for the $a$-nonnegative region.

To state the results, let $S_{p}\left(\mathbb{R}^{N}\right)$ be the best embedding constant from $H^{1}\left(\mathbb{R}^{N}\right)$ into $L^{p}\left(\mathbb{R}^{N}\right)$, i.e.,

$$
S_{p}\left(\mathbb{R}^{N}\right)=\inf _{u \in H^{1}\left(\mathbb{R}^{N}\right) \backslash\{0\}} \frac{\int_{\mathbb{R}^{N}}|\nabla u|^{2}+u^{2} d x}{\left(\int_{\mathbb{R}^{N}}|u|^{p} d x\right)^{\frac{2}{p}}} .
$$

In the theorems stated below, we assume $N \geq 3$. Results for $N=1$ and $N=2$ will be given in Section 7.

THEOREM 1.1 (Best Constants and Nonexistence of Extremal Functions)

(i) $S(a, b)$ is continuous in the full parameter domain (1.2).

(ii) For $b=a+1$, we have $S(a, a+1)=\left(\frac{N-2-2 a}{2}\right)^{2}$, and $S(a, a+1)$ is not achieved.

(iii) For $a<0$ and $b=a$, we have $S(a, a)=S(0,0)$ (the best Sobolev constant), and $S(a, a)$ is not achieved.

THEOREM 1.2 (Best Constants and Existence of Extremal Functions)

(i) For $a<b<a+1, S(a, b)$ is always achieved.

(ii) For $b-a \in(0,1)$ fixed, as $a \rightarrow-\infty, S(a, b)$ is strictly increasing, and

$$
S(a, b)=\left(\frac{N-2-2 a}{2}\right)^{2(b-a)}\left[S_{p}\left(\mathbb{R}^{N}\right)+o(1)\right] .
$$

THEOREM 1.3 (Symmetry Breaking) (i) There is $a_{0} \leq 0$ and a function $h(a)$ defined for $a \leq a_{0}$, satisfying $h\left(a_{0}\right)=a_{0}, a<h(a)<a+1$ for $a<a_{0}$, and $a+1-h(a) \rightarrow 0$ as $-a \rightarrow \infty$, such that for any $(a, b)$ satisfying $a<a_{0}$ and $a<b<h(a)$, the minimizer for $S(a, b)$ is nonradial. 
(ii) There is an open subset $H$ inside the a-negative region containing the set $\left\{(a, a) \in \mathbb{R}^{2}: a<0\right\}$ such that for any $(a, b) \in H$ with $a<b$, the minimizer for $S(a, b)$ is nonradial.

Though the minimizers may be nonradial, we still have the following:

THEOREM 1.4 (Symmetry Property) For $a \leq b<a+1$, any bound state solution $u$ of (1.8) in $\mathscr{D}_{a}^{1,2}$ satisfying $u(x)>0$ for $x \in \mathbb{R}^{N} \backslash\{0\}$, possibly after a dilation $u(x) \rightarrow \tau^{(N-2-2 a) / 2} u(\tau x)$, satisfies the "modified inversion" symmetry:

$$
u\left(\frac{x}{|x|^{2}}\right)=|x|^{N-2-2 a} u(x) .
$$

Moreover, writing $|x|=e^{-t}$ and $\theta=x /|x|$, we have that for fixed $\theta$,

$$
e^{-\frac{N-2-2 a}{2} t} u\left(e^{-t} \theta\right)
$$

is even in $t$ and monotonically decreasing in $t$ for $t>0$.

REMARK 1.5 Some comments are in order here.

1. In Theorems 1.1 and 1.2, we have given the best constants for $(a, b)$ on the "boundary" of the $a$-negative region. Since $S(a, b)$ is continuous, we also obtain estimates for $S(a, b)$ near the boundary of the parameter domain. From Theorems 1.2 and 1.3, there are no closed-form minimizers, so it seems to be very difficult to examine the best constants in the interior of the region.

2. For a special case $b=0,-1<a<0$, the existence of a minimizer was given in [7] by using a quite different method.

3. In the case $b=a$, we have $p=2^{*}$, the critical Sobolev exponent. The situation is quite delicate since for $a \geq 0, S(a, a)$ is strictly decreasing in $a$ and is solvable as we mentioned above [11, 25], and for $a<0$, we have $S(a, a)=S(0,0)$ and the nonexistence result in Theorem 1.1.

4. The results in (i) and (ii) of Theorem 1.3 overlap, but neither implies the other. The importance of (ii) is that symmetry breaking occurs for all $a<0$ if $b$ is sufficiently close to $a$.

5. For Theorem 1.3(i), $a_{0}$ and $h(a)$ will be given explicitly in the proof in Section 6.

Our approach to the problem in this paper is quite different from that used in the quoted previous papers ( worked directly in $\mathscr{D}_{a}^{1,2}\left(\mathbb{R}^{N}\right)$, and we shall take a detour to convert the problem to an equivalent one defined on $H^{1}\left(\mathbb{R} \times \mathbb{S}^{N-1}\right)$. While taking advantage of the two formulations, we shall work mainly with the equivalent one on $H^{1}\left(\mathbb{R} \times \mathbb{S}^{N-1}\right)$. The reformulation enables us to make use of a combination of analytical tools such as a compactness argument, rescaling, the concentration compactness principle, bifurcation analysis, the moving plane method, etc. Moreover, our approach also gives a different proof of inequalities (1.1) (see Remark 2.4).

The organization of the paper is as follows. In Section 2, we shall introduce a transformation that transforms our problem in $\mathbb{R}^{N}$ to one on the space $\mathbb{R} \times \mathbb{S}^{N-1}$ on which we have a family of inequalities corresponding to (1.1) and an Euler 
equation corresponding to (1.8). The two problems will be shown to be equivalent, and we shall mainly work on the transformed one on $\mathbb{R} \times \mathbb{S}^{N-1}$. The advantage in working on the latter is that the equation is an autonomous one and is defined in $H^{1}\left(\mathbb{R} \times \mathbb{S}^{N-1}\right)$. Radial solutions (as we shall see, the only bound state radial solutions are the ground state solutions in the radially symmetric class) will be examined completely and their energy levels will be computed so that some comparison arguments can be done later. In Section 3, we prove Theorem 1.1, first establishing the continuity of $S(a, b)$ in $(a, b)$ and then giving the nonexistence result for the case $b=a$ with a combination of continuity and comparison arguments. In Section 4, the existence of a minimizer for the case $a<b<a+1$ will be given by using a compactness argument; an asymptotic estimate for $S(a, b)$ as $a \rightarrow-\infty$ will be given using a concentration compactness principle. In Section 5, we establish the symmetry-breaking result (Theorem 1.3). First a bifurcation analysis will be done to claim the symmetry breaking for $a$ away from 0 . For $a$ close to 0 it is much subtler, and some continuity and comparison arguments will be employed. Section 6 is devoted to establishing the modified inversion symmetry (up to a dilation) for all bound state solutions of (1.8) by using the moving plane method. In Section 7, we treat the cases $N=1$ and $N=2$. For $N=1$ we have a complete solution for the problem including the identification of all bound state solutions. Finally, in Section 8 , we state results for a related problem that can be solved using our results for (1.8), and we also point out some related open questions in Section 9.

\section{An Equivalent Problem and Some Preliminaries}

In this section, we start by introducing a family of transformations that will transform our original problem to one defined on a cylinder $\mathbb{R} \times \mathbb{S}^{N-1}$. The two problems will be shown to be equivalent in a sense that will be precisely specified. Then some preliminary results on the radial solutions will be given.

\subsection{Equivalent Problems on $\mathbb{R} \times \mathbb{S}^{N-1}$}

To problem (1.1) and equation (1.8) on $\mathbb{R}^{N}$ we shall derive an equivalent minimization problem and corresponding Euler equation on $\mathbb{R} \times \mathbb{S}^{N-1}$. We shall use the notation $\mathcal{C}=\mathbb{R} \times \mathbb{S}^{N-1}$. While working on both problems to take advantage of the two formulations, we shall get most of our results on the cylinder $\mathcal{C}$. For integrals over a domain included in $\mathcal{C}$, by $d \mu$ we denote the volume element on $\mathcal{C}$. Also, by $|\nabla u|^{2}$ we understand $g^{i j} u_{i} u_{j}$ and $\left(g^{i j}\right)$ are the components of the inverse matrix to the metric induced from $\mathbb{R}^{N+1}$. For points on $\mathcal{C}$ we use either the notation $y$ to identify a point in $\mathbb{R}^{N+1}$ or $(t, \theta)$ to identify a point in $\mathbb{R} \times \mathbb{S}^{N-1}$.

To $u$, a smooth function with compact support in $\mathbb{R}^{N} \backslash\{0\}$, we associate $v$, a smooth function with compact support on $\mathcal{C}$, by the transformation

$$
u(x)=|x|^{-\frac{N-2-2 a}{2}} v\left(-\ln |x|, \frac{x}{|x|}\right) \text {. }
$$


Here for $x \in \mathbb{R}^{N} \backslash\{0\}$, with $t=-\ln |x|$ and $\theta=x /|x|$, we have $(t, \theta) \in \mathcal{C}$.

Let us denote by $L_{b}^{p}\left(\mathbb{R}^{N}\right)=\left\{u: \int_{\mathbb{R}^{N}}|x|^{-b p}|u|^{p} d x<\infty\right\}$ the weighted $L^{p}$ space. We need the following lemma.

LEMMA 2.1 For $a<\frac{N-2}{2}, a \leq b \leq a+1$, and $p=\frac{2 N}{N-2+2(b-a)}$, it holds that

$$
\mathscr{D}_{a}^{1,2}\left(\mathbb{R}^{N}\right)=\overline{C_{0}^{\infty}\left(\mathbb{R}^{N} \backslash\{0\}\right)}{ }^{\|\cdot\|},
$$

where $\|\cdot\|$ is the norm in $\mathscr{D}_{a}^{1,2}\left(\mathbb{R}^{N}\right)$ given by (1.5). Moreover, $L_{b}^{p}\left(\mathbb{R}^{N}\right)$ is also given by the completion of $C_{0}^{\infty}\left(\mathbb{R}^{N} \backslash\{0\}\right)$ under its norm.

Proof: By the definition of $\mathscr{D}_{a}^{1,2}\left(\mathbb{R}^{N}\right)$, it suffices to show

$$
\left.C_{0}^{\infty}\left(\mathbb{R}^{N}\right) \subset \overline{C_{0}^{\infty}\left(\mathbb{R}^{N} \backslash\{0\}\right)}\right)^{\|\cdot\|} .
$$

Let $\rho(t)$ be a cutoff function that is 1 for $t \geq 2$ and 0 for $0<t \leq 1$. For a fixed $u \in C_{0}^{\infty}\left(\mathbb{R}^{N}\right)$, we define $u_{\varepsilon}(x)=\rho(|x| / \varepsilon) u(x) \in C_{0}^{\infty}\left(\mathbb{R}^{N} \backslash\{0\}\right)$. Then it is easy to check that $\left\|u_{\varepsilon}-u\right\| \rightarrow 0$ as $\varepsilon \rightarrow 0$. The second part is similar.

Now for $u \in C_{0}^{\infty}\left(\mathbb{R}^{N} \backslash\{0\}\right)$, by a direct computation we have

$$
\int_{\mathbb{R}^{N}}|x|^{-2 a}|\nabla u|^{2}(x) d x=\int_{\mathbb{R}^{N}}|x|^{-N}\left(\left|\nabla_{\theta} v\right|^{2}+\left(v_{t}+\frac{N-2-2 a}{2} v\right)^{2}\right) d x ;
$$

therefore

$$
\begin{aligned}
\int_{\mathbb{R}^{N}}|x|^{-2 a}|\nabla u|^{2}(x) d x & =\int_{\mathcal{C}}\left|\nabla_{\theta} v\right|^{2}+\left(v_{t}+\frac{N-2-2 a}{2} v\right)^{2} d \mu \\
& =\int_{\mathcal{C}}\left|\nabla_{\theta} v\right|^{2}+v_{t}^{2}+\left(\frac{N-2-2 a}{2}\right)^{2} v^{2} d \mu .
\end{aligned}
$$

Also,

$$
\int_{\mathbb{R}^{N}}|x|^{-b p} u^{p}(x) d x=\int_{\mathbb{R}^{N}}|x|^{-N} v^{p} d x=\int_{\mathcal{C}} v^{p} d \mu .
$$

From these and Lemma 2.1, we immediately have the following:

PROPOSITION 2.2 The mapping given in (2.1) is a Hilbert space isomorphism from $\mathscr{D}_{a}^{1,2}\left(\mathbb{R}^{N}\right)$ to $H^{1}(\mathcal{C})$ where the inner product on $H^{1}(\mathcal{C})$ is

$$
(v, w)=\int_{\mathcal{C}} \nabla v \cdot \nabla w+\left(\frac{N-2-2 a}{2}\right)^{2} v w d \mu .
$$

Now we define an energy functional on $H^{1}(\mathcal{C})$ :

$$
F_{a, b}(v)=\frac{\int_{\mathcal{C}}\left|\nabla_{\theta} v\right|^{2}+v_{t}^{2}+\left(\frac{N-2-2 a}{2}\right)^{2} v^{2} d \mu}{\left(\int_{\mathcal{C}}|v|^{p} d \mu\right)^{2 / p}} .
$$


If $u \in \mathscr{D}_{a}^{1,2}\left(\mathbb{R}^{N}\right)$ and $v \in H^{1}(\mathcal{C})$ are related through (2.1), then

$$
E_{a, b}(u)=F_{a, b}(v) .
$$

Moreover, if $u$ is a solution of (1.8), then $v$ satisfies

$$
-v_{t t}-\Delta_{\theta} v+\left(\frac{N-2-2 a}{2}\right)^{2} v=v^{p-1}, \quad v>0, \quad \text { on } C
$$

where $t=-\ln |x|$ and $\Delta_{\theta}$ is the Laplace operator on the $(N-1)$-sphere. We collect these observations in the following:

Proposition 2.3 With $a, b$, and $p$ satisfying (1.2), we have

(i) If $u \in \mathscr{D}_{a}^{1,2}$ and $v \in H^{1}(\mathcal{C})$ are related through $(2.1)$, then $E_{a, b}(u)=$ $F_{a, b}(v)$.

(ii) For $S(a, b)$ defined in (1.6), it holds $S(a, b)=\inf _{H^{1}(\mathcal{C}) \backslash\{0\}} F_{a, b}(v)$.

(iii) Solutions of (1.8) and (2.3) are in one-to-one correspondence, being related through (2.1).

REMARK 2.4 Our approach here gives a new independent proof of the CaffarelliKohn-Nirenberg inequalities for the considered parameters, because by the classical Sobolev embeddings from $H^{1}(\mathcal{C})$ into $L^{p}(\mathcal{C}), F_{a, b}(v)$ has a positive lower bound on $H^{1}(\mathcal{C})$ and the transformation (2.1) gives the desired inequalities on $\mathcal{D}_{a}^{1,2}\left(\mathbb{R}^{N}\right)$.

REMARK 2.5 As motivation, we mention that transformations of similar nature to (2.1) have been used in the past to study radial solutions (e.g., [18]), which link two ODEs. For PDEs, this was used recently for the Yamabe problem $(a=$ $b=0)$ in [17]. In this paper we have developed the full-blown version of the transformations to deal with solutions of PDEs (1.8), and furthermore we have established the equivalence of the function spaces involved.

\subsection{Invariance of the Problem (1.8)}

In order to study the symmetry property of solutions, we examine the invariance of the problem under the transformation (2.1). As in the case of the Yamabe problem $(a=b=0)$, the group of transformations that leaves problem (1.8) invariant is noncompact. The group of translations in $\mathbb{R}^{N}$ is a symmetry group for (1.8) only in the case $a=b=0$. On the other hand, the dilations

$$
u_{\tau}(x)=\tau^{\frac{N-2-2 a}{2}} u(\tau x), \quad \tau>0,
$$

leave the problem invariant for all $a$ and $b$; i.e., if $u$ is a solution of (1.8), so is $u_{\tau}$. This still holds for $N=2$ and $N=1$, but for $N=1$ the situation is a bit different and there is a two-parameter family of dilations (see (7.3)). The group that leaves (2.3) invariant, corresponding to dilations in $\mathbb{R}^{N}$, is the group of translations in the $t$-direction. If $v$ and $v_{\tau}$ in $H^{1}(\mathcal{C})$ are related to $u$ and $u_{\tau}$ in $\mathscr{D}_{a}^{1,2}\left(\mathbb{R}^{N}\right)$ through $(2.1)$, then

$$
v_{\tau}(t, \theta)=v(t-\ln \tau, \theta)
$$


Finally, the following modified inversion invariance of (1.8),

$$
\bar{u}(x)=|x|^{-(N-2-2 a)} u\left(\frac{x}{|x|^{2}}\right),
$$

translates on the cylinder to the following obvious symmetry of (2.3),

$$
\bar{v}(t, \theta)=v(-t, \theta) .
$$

\subsection{Radial Solutions} fine

Let $\mathscr{D}_{a, R}^{1,2}\left(\mathbb{R}^{N}\right)$ be the subspace of $\mathscr{D}_{a}^{1,2}\left(\mathbb{R}^{N}\right)$ consisting of radial functions. De-

$$
R(a, b)=\inf _{u \in D_{a, R}^{1,2}\left(\mathbb{R}^{N}\right) \backslash\{0\}} E_{a, b}(u) .
$$

By Proposition 2.3(i) we also have

$$
R(a, b)=\inf _{u \in H_{R}^{1}(\mathcal{C}) \backslash\{0\}} F_{a, b}(u),
$$

where $H_{R}^{1}(\mathcal{C})$ consists of functions independent of $\theta$. We shall find the exact value of $R(a, b)$ and the exact form of the radial solutions that achieve these constants when $a \leq b<a+1$. We remark here that our method applies for the $a$-nonnegative region also and in fact gives a new approach for the $a$-nonnegative region; the results we get agree with [11] and [18] in this region.

In order to study the radial solutions of (1.8), we shall need the exact form of particular positive solutions for the following nonlinear second-order ODE:

$$
-v_{t t}+\lambda^{2} v=v^{p-1}, \quad v>0, \quad \text { in } \mathbb{R}
$$

with $p>2$. The problem can be associated to the Hamiltonian system

$$
\frac{d}{d t} v=w, \quad \frac{d}{d t} w=\lambda^{2} v-v^{p-1} .
$$

We have the Hamiltonian

$$
H(v, w)=\frac{1}{2} w^{2}-\frac{\lambda^{2}}{2} v^{2}+\frac{1}{p} v^{p} .
$$

All solutions correspond to level curves of $H(v, w)$. Up to translations, there is only one homoclinic solution $v$ that is on the level $H(v, w)=0$. The levels below this one will give $v$ positive, periodic, and bounded away from zero. For the levels above, $v$ changes sign so we lose positivity. The only positive solutions that are in $H^{1}(\mathbb{R})$ are translates of

$$
v(t)=\left(\frac{\lambda^{2} p}{2}\right)^{\frac{1}{p-2}}\left(\cosh \left(\frac{p-2}{2} \lambda t\right)\right)^{-\frac{2}{p-2}} .
$$


A direct calculation gives that for the $v$ above,

$$
\frac{\int_{\mathbb{R}} v_{t}^{2}+\lambda^{2} v^{2} d t}{\left(\int_{\mathbb{R}} v^{p} d t\right)^{2 / p}}=2 p \frac{\lambda^{(p+2) / p}}{(p-2)^{(p-2) / p}}\left(\frac{\Gamma^{2}\left(\frac{p}{p-2}\right)}{\Gamma\left(\frac{2 p}{p-2}\right)}\right)^{\frac{p-2}{p}} .
$$

Now, when searching for radial solutions, equation (2.3) becomes

$$
v_{t t}-\left(\frac{N-2-2 a}{2}\right)^{2} v+v^{p-1}=0, \quad v>0, \quad \text { on } \mathbb{R},
$$

which corresponds to equation (2.7) with $\lambda=\frac{N-2-2 a}{2}$. According to (2.8), the homoclinic solutions of (2.10) are translates of

$$
\begin{aligned}
v(t)= & \left(\frac{N(N-2-2 a)^{2}}{4(N-2(1+a-b))}\right)^{\frac{N-2(1+a-b)}{4(1+a-b)}} \\
& \left(\cosh \frac{(N-2-2 a)(1+a-b)}{N-2(1+a-b)} t\right)^{-\frac{N-2(1+a-b)}{2(1+a-b)}} .
\end{aligned}
$$

The radial solution in $\mathbb{R}^{N}$ for (1.8) corresponding to this $v$ is

$$
u(x)=\left(\frac{N(N-2-2 a)^{2}}{N-2(1+a-b)}\right)^{\frac{N-2(1+a-b)}{4(1+a-b)}} \frac{1}{\left(1+|x|^{\frac{2(N-2-2 a)(1+a-b)}{N-2(1+a-b)}}\right)^{\frac{N-2(1+a-b)}{2(1+a-b)}}}
$$

All radial solutions in $\mathbb{R}^{N}$ for (1.8) are dilations of this $u$. Note that by substituting in (2.9)

$$
\lambda=\frac{N-2-2 a}{2} \quad \text { and } \quad p=\frac{2 N}{N-2(1+a-b)},
$$

we estimate the energy of any radial solution in $H^{1}(\mathcal{C})$,

$$
\begin{gathered}
R(a, b)=E_{a, b}(u)=F_{a, b}(v), \\
R(a, b)=\frac{N \omega_{N-1}^{\frac{2(1+a-b)}{N}}(N-2-2 a)^{\frac{2(N-(1+a-b))}{N}}}{2^{\frac{2(1+a-b)}{N}}(N-2(1+a-b))^{\frac{N-2(1+a-b)}{N}}(1+a-b)^{\frac{2(1+a-b)}{N}}} \\
\left(\frac{\Gamma^{2}\left(\frac{N}{2(1+a-b)}\right)}{\Gamma\left(\frac{N}{1+a-b}\right)}\right)^{\frac{2(1+a-b)}{N}} .
\end{gathered}
$$

PROPOSITION 2.6 Up to a dilation (2.4), all radial solutions of (1.8) are explicitly given in (2.12), and $R(a, b)$ is given in (2.13).

REMARK 2.7 In the case $a>0$, this is the best constant as found in [11], i.e., $R(a, b)=S(a, b)$. Also, for $a=0$ and $0 \leq b<1$, it is the best constant found by Lieb in [18]. In the case $a \geq 0$, up to a dilation (and also a translation in the case $a=b=0),(2.12)$ gives all bound state solutions of (1.8) that achieve equality in the Caffarelli-Kohn-Nirenberg inequality (see $[11,18]$ ). 


\section{Best Constants and Nonexistence of Extremal Functions}

To prove Theorem 1.1(i), we need a couple of lemmas.

LeMmA 3.1 Let $a_{0}<\frac{N-2}{2}, a_{0} \leq b_{0} \leq a_{0}+1$. Then

$$
\limsup _{(a, b) \rightarrow\left(a_{0}, b_{0}\right)} S(a, b) \leq S\left(a_{0}, b_{0}\right) .
$$

Proof: For any $\varepsilon>0$, there is a nonnegative function $v \in C_{0}^{\infty}(\mathcal{C})$ such that

$$
F_{a_{0}, b_{0}}(v) \leq S\left(a_{0}, b_{0}\right)+\frac{\varepsilon}{2} .
$$

Note that as $(a, b) \rightarrow\left(a_{0}, b_{0}\right), v^{p}(x) \rightarrow v^{p_{0}}(x)$ for all $x$. For any $p \in\left[2,2^{*}\right]$, $v^{p}(x) \leq w(x)$ where $w(x)=v^{2}(x)$ if $v(x)<1$ and $w(x)=v^{2^{*}}(x)$ if $v(x) \geq 1$. Clearly $w$ is integrable; therefore by the dominated convergence theorem we have

$$
\lim _{(a, b) \rightarrow\left(a_{0}, b_{0}\right)} \int_{\mathcal{C}} v^{p} d \mu=\int_{\mathcal{C}} v^{p_{0}} d \mu .
$$

From this, and because $\lambda$ is continuous in $a$, we get there is $\delta>0$ such that $\left|(a, b)-\left(a_{0}, b_{0}\right)\right|<\delta$ implies

$$
S(a, b) \leq F_{a, b}(v) \leq F_{a_{0}, b_{0}}(v)+\frac{\varepsilon}{2} \leq S\left(a_{0}, b_{0}\right)+\varepsilon .
$$

Let $\varepsilon \rightarrow 0$.

LEMMA 3.2 Let $\left(p_{n}\right) \subset\left[2,2^{*}\right]$ be a sequence convergent to $p$. If a sequence $\left(u_{n}\right)$ is uniformly bounded by $M$ in $H^{1}(\mathcal{C})$, then

(i) if $p \in\left(2,2^{*}\right)$, we have

$$
\left.\lim _{n \rightarrow \infty} \int_{\mathcal{C}}|| u_{n}\right|^{p_{n}}-\left|u_{n}\right|^{p} \mid d \mu=0 ;
$$

(ii) if $p=2$ or $p=2^{*}$, we have

$$
\limsup _{n \rightarrow \infty} \int_{\mathcal{C}}\left(\left|u_{n}\right|^{p_{n}}-\left|u_{n}\right|^{p}\right) d \mu \leq 0 .
$$

PROOF: We first prove (i). By the mean value theorem, there are functions $\xi_{n}$ defined on $\mathcal{C}$ with values between $p_{n}$ and $p$ such that

$$
\left.\int_{\mathcal{C}}|| u_{n}\right|^{p_{n}}-\left|u_{n}\right|^{p}\left|d \mu=\int_{\mathcal{C}}\right| \ln \left|u_{n}\right|\left|u_{n}\right|^{\xi_{n}(x)}\left(p_{n}-p\right) \mid d \mu .
$$


Since $p \in\left(2,2^{*}\right)$, let $\varepsilon>0$ such that $[p-\varepsilon, p+\varepsilon] \subset\left(2,2^{*}\right)$. Let $n_{\varepsilon}$ be such that for $n \geq n_{\varepsilon}$ we have $\left|p_{n}-p\right|<\varepsilon$; therefore

$$
\begin{aligned}
\left.\int_{\mathcal{C}}|| u_{n}\right|^{p_{n}}-\left|u_{n}\right|^{p} \mid d \mu \leq & \\
& \left|p_{n}-p\right|\left(\int_{\left|u_{n}\right|>1} \ln \left|u_{n}\right|\left|u_{n}\right|^{p+\varepsilon} d \mu+\int_{0<\left|u_{n}\right|<1} \ln \frac{1}{\left|u_{n}\right|}\left|u_{n}\right|^{p-\varepsilon} d \mu\right) .
\end{aligned}
$$

The key now is to show that the two integrals on the right-hand side are bounded as $n \rightarrow \infty$. There is a constant $C$ depending only on $p$ such that

$$
\ln u \leq C u^{2^{*}-p-\varepsilon} \quad \text { for all } u>1
$$

and

With

$$
\ln \frac{1}{u} \leq \frac{C}{u^{p-\varepsilon-2}} \quad \text { for all } 0<u<1
$$

we obtain

$$
S_{p}(\mathcal{C})=\inf _{u \in H^{1}(\mathcal{C}) \backslash\{0\}} \frac{\int_{\mathcal{C}}|\nabla u|^{2}+u^{2} d \mu}{\left(\int_{\mathcal{C}}|u|^{p} d \mu\right)^{2 / p}},
$$

$$
\int_{\left|u_{n}\right|>1} \ln \left|u_{n}\right|\left|u_{n}\right|^{p+\varepsilon} d \mu \leq C \int_{\left|u_{n}\right|>1}\left|u_{n}\right|^{2^{*}} d \mu \leq C\left(\frac{M}{S_{2^{*}}(\mathcal{C})}\right)^{\frac{2^{*}}{2}} .
$$

We also have that

$$
\int_{0<\left|u_{n}\right|<1} \ln \frac{1}{\left|u_{n}\right|}\left|u_{n}\right|^{p-\varepsilon} d \mu \leq C \int_{0<\left|u_{n}\right|<1}\left|u_{n}\right|^{2} d \mu \leq C \frac{M}{S_{2}(\mathcal{C})} .
$$

This concludes the proof of (i).

For part (ii), we use the same method as above after we make the estimates as follows: For $p=2$,

$$
\int_{\mathcal{C}}\left|u_{n}\right|^{p_{n}}-\left|u_{n}\right|^{2} d \mu \leq \int_{\left|u_{n}\right|>1}\left|u_{n}\right|^{p_{n}}-\left|u_{n}\right|^{2} d \mu,
$$

and for $p=2^{*}$,

$$
\int_{\mathcal{C}}\left|u_{n}\right|^{p_{n}}-\left|u_{n}\right|^{2^{*}} d \mu \leq \int_{0<\left|u_{n}\right|<1}\left|u_{n}\right|^{p_{n}}-\left|u_{n}\right|^{2^{*}} d \mu .
$$

REMARK 3.3 In the cases $p=2$ or $p=2^{*}$, one can construct sequences $\left(u_{n}\right)$ bounded in $H^{1}(\mathcal{C})$ such that $\left|u_{n}\right|_{L^{p}}=1$ for all $n$, while $\left|u_{n}\right|_{L^{p_{n}}} \rightarrow 0$ as $p_{n} \rightarrow p$. Thus Lemma 3.2(ii) is sharp. 
Proof of TheOrem 1.1(i): According to Lemma 3.1, it suffices to show that

$$
\liminf _{(a, b) \rightarrow\left(a_{0}, b_{0}\right)} S(a, b) \geq S\left(a_{0}, b_{0}\right) .
$$

Assume there is a sequence $\left(a_{n}, b_{n}\right) \rightarrow\left(a_{0}, b_{0}\right)$ such that

$$
\lim _{n \rightarrow \infty} S\left(a_{n}, b_{n}\right)<S\left(a_{0}, b_{0}\right) \text {. }
$$

Then there are $\varepsilon>0$ and functions $\left(v_{n}\right) \subset H^{1}(\mathcal{C})$ such that

$$
\int_{\mathcal{C}}\left|v_{n}\right|^{p_{n}} d \mu=1
$$

and

$$
S\left(a_{0}, b_{0}\right)-\varepsilon \geq F_{a_{n}, b_{n}}\left(v_{n}\right) .
$$

Clearly, $\left(v_{n}\right)$ is bounded in $H^{1}(\mathcal{C})$. From Lemma 3.2, we get

$$
F_{a_{n}, b_{n}}\left(v_{n}\right)+o(1) \geq F_{a_{0}, b_{0}}\left(v_{n}\right) \geq S\left(a_{0}, b_{0}\right) .
$$

This and (3.1) give the desired contradiction.

REMARK 3.4 A similar proof shows that $R(a, b)$ is continuous in $(a, b)$ in the full parameter region, including the upper boundary $\{b=a+1\}$ for which no radial solutions exist.

Proof of TheOREM 1.1(ii): Clearly, $F_{a, a+1}(v) \geq((N-2-2 a) / 2)^{2}$ for all $v \in H^{1}(\mathcal{C})$. On the other hand, one can easily construct a sequence $\left(v_{n}\right) \subset H^{1}(\mathcal{C})$ of radial functions such that $F_{a, a+1}\left(v_{n}\right) \rightarrow((N-2-2 a) / 2)^{2}$. Therefore,

$$
S(a, a+1)=\left(\frac{N-2-2 a}{2}\right)^{2} .
$$

For nonexistence of minimizers, one notes that for $\lambda \geq 1$, the equation

$$
-\Delta v+\lambda^{2} v=v
$$

has no nonzero solution in $H^{1}(\mathcal{C})$. For $0<\lambda<1$, i.e., $(N-4) / 2<a<$ $(N-2) / 2$, assume that $S(a, a+1)$ is achieved by some function $v \in H^{1}(\mathcal{C})$. By the maximum principle, $v>0$ everywhere. Denote by $f(t)$ the average of $v$ on the spheres $t=$ const. Then $f$ is a positive function in $H^{1}(\mathbb{R})$ and satisfies the ODE

$$
-f_{t t}+\lambda^{2} f=f
$$

The only nonnegative solution is $f \equiv 0$. Therefore for all $a<\frac{N-2}{2}$, the infimum $S(a, a+1)$ is not achieved.

Proof of TheOREM 1.1(iii): The case $a=b=0$ is well known (the Yamabe problem in $\left.\mathbb{R}^{N}\right)$. In this case, the minimizer $S(0,0)$ is achieved only by functions

$$
U_{\mu, y}(x)=C \frac{\mu^{(N-2) / 2}}{\left(\mu^{2}+|x-y|^{2}\right)^{(N-2) / 2}}, \quad \mu>0, \quad y \in \mathbb{R}^{N} .
$$


Note that for $a \in(-N / 2,(N-2) / 2), U_{\mu, y} \in \mathcal{D}_{a}^{1,2}$. For $y \neq 0$ by a direct computation we get for $a \in(-N / 2,(N-2) / 2)$

$$
S(0,0)=\lim _{\mu \rightarrow 0} E_{a, a}\left(U_{\mu, y}\right) .
$$

Due to this fact one concludes that for $a \in(-N / 2,(N-2) / 2)$,

$$
S(a, a) \leq S(0,0) .
$$

On the other hand, by the expression (2.2), for any $v \in H^{1}(\mathcal{C}) \backslash\{0\}, F_{a, a}(v)>$ $F_{0,0}(v) \geq S(0,0)$. Hence, $S(a, a)=S(0,0)$ for all $a \in(-N / 2,0)$. Next, we fix $a_{1} \in(-N / 2,0)$. For any $a \leq-N / 2$ fixed and any $\varepsilon>0$, there is $v \in H^{1}(\mathcal{C})$ such that

$$
F_{a_{1}, a_{1}}(v) \leq S(0,0)+\frac{\varepsilon}{2\left(\lambda(a)^{2}-\lambda\left(a_{1}\right)^{2}\right)\left(\lambda\left(a_{1}\right)^{2}-\lambda(0)^{2}\right)},
$$

where $\lambda(a)=(N-2-2 a) / 2$. Together with $S(0,0) \leq F_{0,0}(v) \leq F_{a_{1}, a_{1}}(v)$, we conclude

$$
\frac{\int_{\mathcal{C}} v^{2} d \mu}{\left(\int_{\mathcal{C}}|v|^{2^{*}} d \mu\right)^{2 / 2^{*}}} \leq \frac{\varepsilon}{2\left(\lambda(a)^{2}-\lambda\left(a_{1}\right)^{2}\right)}
$$

Then

$$
F_{a, a}(v)=F_{a_{1}, a_{1}}(v)+\left(\lambda(a)^{2}-\lambda\left(a_{1}\right)^{2}\right) \frac{\int_{\mathcal{C}} v^{2} d \mu}{\left(\int_{\mathcal{C}}|v|^{2^{*}} d \mu\right)^{2 / 2^{*}}} \leq S(0,0)+\varepsilon .
$$

That is, $S(a, a)=S(0,0)$ for all $a \leq 0$.

Next we show $S(a, a)$ is not achieved for $a<0$. If the conclusion is not true, for some $a<0$ and $v \in H^{1}(\mathcal{C})$ we get $S(a, a)=F_{a, a}(v)$. But using $F_{a, a}(v)>F_{0,0}(v) \geq S(0,0)$, we get a contradiction to $S(a, a)=S(0,0)$.

\section{Best Constants and Existence of Extremal Functions}

In this section we prove the existence of a minimizer for $a<0$ and $a<b<$ $a+1$. We also give an asymptotic estimate of $S(a, b)$ as $-a \rightarrow \infty$, while $b-a \in$ $(0,1)$ is a fixed constant.

We shall need the following lemma. It is analogous to a result on $\mathbb{R}^{N}$ due to P. L. Lions [21]. The proof is similar to the proof of lemma 1.21 in [26]. We omit the proof here.

LEMMA 4.1 Let $r>0$ and $2 \leq q<2^{*}$. If $\left(w_{n}\right)$ is bounded in $H^{1}(\mathcal{C})$ and if

$$
\sup _{y \in \mathcal{C}} \int_{B_{r}(y) \cap \mathcal{C}}\left|w_{n}\right|^{q} d \mu \rightarrow 0 \quad \text { as } n \rightarrow \infty,
$$

then $w_{n} \rightarrow 0$ in $L^{p}(\mathcal{C})$ for $2<p<2^{*}$. Here $B_{r}(y)$ denotes the ball in $\mathbb{R}^{N+1}$ with radius $r$ centered at $y$. 
Proof of TheOrem 1.2(i): Let $a<0$ and $a<b<a+1$ be fixed. Consider a minimizing sequence $\left(w_{n}\right) \subset H^{1}(\mathcal{C})$ such that

$$
\int_{\mathcal{C}}\left|w_{n}\right|^{p} d \mu=1 \text { for all } n \geq 1
$$

and

$$
\int_{\mathcal{C}}\left|\nabla w_{n}\right|^{2}+\left(\frac{N-2-a}{2}\right)^{2} w_{n}^{2} d \mu \rightarrow S(a, b) \quad \text { as } n \rightarrow \infty .
$$

According to Lemma 4.1,

$$
\delta=\liminf _{n \rightarrow \infty}\left(\sup _{y \in \mathcal{C}} \int_{B_{r}(y) \cap \mathcal{C}} w_{n}^{2} d \mu\right)>0 .
$$

Eventually by passing to a subsequence, we may assume there are $\left(y_{n}\right) \subset \mathcal{C}$ and $y_{0} \in \mathcal{C}$ fixed such that the sequence $v_{n}(x)=w_{n}\left(x-y_{n}\right)$ has the property

$$
\int_{B_{r}\left(y_{0}\right) \cap e}\left|v_{n}\right|^{2} d \mu>\frac{\delta}{2} .
$$

Clearly,

$$
\int_{\mathcal{C}}\left|v_{n}\right|^{p} d \mu=1 \text { for all } n \geq 1
$$

and

$$
\int_{\mathcal{C}}\left|\nabla v_{n}\right|^{2}+\left(\frac{N-2-a}{2}\right)^{2} v_{n}^{2} d \mu \rightarrow S(a, b) \quad \text { as } n \rightarrow \infty .
$$

Without loss of generality we can assume

$$
\begin{array}{ll}
v_{n} \rightarrow v & \text { weakly in } H^{1}(\mathcal{C}), \\
v_{n} \rightarrow v & \text { in } L_{\text {loc }}^{2}(\mathcal{C}), \\
v_{n} \rightarrow v & \text { almost everywhere in } \mathcal{C} .
\end{array}
$$

According to the Brezis-Lieb lemma [3], we have

$$
1=|v|_{L^{p}}^{p}+\lim _{n \rightarrow \infty}\left|v_{n}-v\right|_{L^{p}}^{p} .
$$


Hence

$$
\begin{aligned}
S(a, b)= & \lim _{n \rightarrow \infty} \int_{\mathcal{C}}\left|\nabla v_{n}\right|^{2}+\left(\frac{N-2-a}{2}\right)^{2} v_{n}^{2} d \mu \\
= & \int_{\mathcal{C}}|\nabla v|^{2}+\left(\frac{N-2-a}{2}\right)^{2} v^{2} d \mu \\
& +\lim _{n \rightarrow \infty} \int_{\mathcal{C}}\left|\nabla v_{n}-v\right|^{2}+\left(\frac{N-2-a}{2}\right)^{2}\left(v_{n}-v\right)^{2} d \mu \\
\geq & S(a, b)\left(|v|_{L^{p}}^{2}+\left(1-|v|_{L^{p}}^{p}\right)^{\frac{2}{p}}\right) .
\end{aligned}
$$

Since $v \not \equiv 0$, we obtain $|v|_{L^{p}}=1$, and so

$$
\int_{\mathcal{C}}|\nabla v|^{2}+\left(\frac{N-2-a}{2}\right)^{2} v^{2} d \mu=S(a, b) .
$$

Let $b-a \in(0,1)$ be fixed so that $p \in\left(2,2^{*}\right)$ is also fixed. We shall consider the asymptotic behavior of $S(a, b)$ as $-a \rightarrow \infty$.

Proof of THEOREM 1.2(ii): We use a rescaling argument. Let $h_{\lambda}: \mathbb{R}^{N+1} \rightarrow$ $\mathbb{R}^{N+1}$ be the scaling map $h_{\lambda}(x)=\lambda x$. Denote $\mathcal{C}_{\lambda}=h_{\lambda}(\mathcal{C})$ and for $v \in H^{1}(\mathcal{C})$, define $u \in H^{1}\left(\mathcal{C}_{\lambda}\right)$ by $u(\lambda x)=v(x)$. For definiteness, on $H^{1}\left(\mathcal{C}_{\lambda}\right)$ we use the norm $\|u\|^{2}=\int_{\mathcal{C}_{\lambda}}|\nabla u|^{2}+|u|^{2} d \mu$. We have

$$
\int_{\mathcal{C}}|\nabla v|^{2}+\lambda^{2} v^{2} d \mu=\lambda^{2-N} \int_{\mathcal{C}_{\lambda}}|\nabla u|^{2}+u^{2} d \mu
$$

and

$$
\int_{\mathcal{C}}|v|^{p} d \mu=\lambda^{-N} \int_{\mathcal{C}_{\lambda}}|u|^{p} d \mu
$$

Therefore,

$$
F_{a, b}(v)=\lambda^{2(b-a)} \frac{\int_{\mathcal{C}_{\lambda}}|\nabla u|^{2}+u^{2} d \mu}{\left(\int_{\mathcal{C}_{\lambda}}|u|^{p} d \mu\right)^{\frac{2}{p}}}
$$

Now it suffices to show that

$$
I(\lambda):=\inf _{u \in H^{1}\left(\mathcal{C}_{\lambda}\right) \backslash\{0\}} \frac{\int_{\mathcal{C}_{\lambda}}|\nabla u|^{2}+u^{2} d \mu}{\left(\int_{\mathcal{C}_{\lambda}}|u|^{p} d \mu\right)^{2 / p}} \rightarrow S_{p}\left(\mathbb{R}^{N}\right)
$$

as $\lambda \rightarrow \infty$.

First we have that

$$
\limsup _{\lambda \rightarrow \infty} I(\lambda) \leq S_{p}\left(\mathbb{R}^{N}\right) .
$$


We get this through a cutoff procedure. Let $r>0$; then for fixed $\lambda$ large and $y \in \mathcal{C}_{\lambda}$, we have a projection $\psi=\psi_{y, r, \lambda}$ from $B_{r}(0) \subset \mathbb{R}^{N}$ to $\psi\left(B_{r}(0)\right) \subset \mathcal{C}_{\lambda}$ defined as follows: Identify $\mathbb{R}^{N}$ with the tangent space to $\mathcal{C}_{\lambda}$ at $y \in \mathcal{C}_{\lambda}$, and let $\psi$ to be the projection from $B_{r}(0)$ into $\mathcal{C}_{\lambda}$ along directions parallel to $v_{y}$, the exterior normal to $\mathcal{C}_{\lambda}$ at $y$. Then $\psi$ is a diffeomorphism on its image and for fixed $r$, the Jacobian matrix of $\psi$ tends uniformly to the identity matrix as $\lambda \rightarrow \infty$.

Denote by $w \in H^{1}\left(\mathbb{R}^{N}\right)$ a function with support in $B_{r}(0) \subset \mathbb{R}^{N}$. For $y \in \mathcal{C}_{\lambda}$, let $u_{\lambda}\left(\psi_{y, r, \lambda}(x)\right)=w(x)$ and 0 outside $\psi_{y, r, \lambda}\left(B_{r}(0)\right)$; then

$$
\int_{\mathcal{C}_{\lambda}}\left|\nabla u_{\lambda}\right|^{2}+u_{\lambda}^{2} d \mu=\int_{\mathbb{R}^{N}}|\nabla w|^{2}+w^{2} d x+o(1)
$$

and

$$
\int_{\mathcal{C}_{\lambda}}\left|u_{\lambda}\right|^{p} d \mu=\int_{\mathbb{R}^{N}}|w|^{p} d x+o(1)
$$

where $o(1) \rightarrow 0$ as $\lambda \rightarrow \infty$ uniformly in $y$.

In $\mathbb{R}^{N}$, it is known that the infimum $S_{p}\left(\mathbb{R}^{N}\right)$ is achieved by a positive function $w$, radially symmetric about some point, which satisfies

$$
-\Delta w+w=w^{p-1} \quad \text { in } \mathbb{R}^{N} .
$$

To prove (4.1), let $\varepsilon>0$ and let $r>r_{0}>0$, sufficiently large, so that for a cutoff function $\rho(x)$, which is identically 1 in $B_{r_{0}}(0)$ and 0 outside $B_{r}(0)$, we have

$$
\frac{\int_{\mathbb{R}^{N}}|\nabla(\rho w)|^{2}+(\rho w)^{2} d x}{\left(\int_{\mathbb{R}^{N}}(\rho w)^{p} d x\right)^{2 / p}}<S_{p}\left(\mathbb{R}^{N}\right)+\frac{\varepsilon}{2} .
$$

Then from (4.2) and (4.3), there is $\lambda$ large enough such that when we consider

$$
u(x)=(\rho w)\left(\psi^{-1}(x)\right) \in H^{1}\left(\mathcal{C}_{\lambda}\right),
$$

we get

$$
\frac{\int_{\mathcal{C}_{\lambda}}|\nabla u|^{2}+u^{2} d \mu}{\left(\int_{\mathcal{C}_{\lambda}} u^{p} d \mu\right)^{2 / p}}<\frac{\int_{\mathbb{R}^{N}}|\nabla(\rho w)|^{2}+(\rho w)^{2} d x}{\left(\int_{\mathbb{R}^{N}}(\rho w)^{p} d x\right)^{2 / p}}+\frac{\varepsilon}{2} .
$$

From the two inequalities we conclude that

$$
I(\lambda) \leq \frac{\int_{\mathcal{C}_{\lambda}}|\nabla u|^{2}+u^{2} d \mu}{\left(\int_{\mathcal{C}_{\lambda}} u^{p} d \mu\right)^{2 / p}}<S_{p}\left(\mathbb{R}^{N}\right)+\varepsilon .
$$

Therefore,

$$
\limsup _{\lambda \rightarrow \infty} I(\lambda) \leq S_{p}\left(\mathbb{R}^{N}\right)+\varepsilon
$$

We now prove

$$
\liminf _{\lambda \rightarrow \infty} I(\lambda) \geq S_{p}\left(\mathbb{R}^{N}\right) .
$$


If (4.4) does not hold, there are $\varepsilon_{0}>0$ and a sequence $\left(\lambda_{n}\right)$ which tends to $\infty$ such that

$$
I_{0}:=\lim _{n \rightarrow \infty} I\left(\lambda_{n}\right) \leq S_{p}\left(\mathbb{R}^{N}\right)-\varepsilon_{0} .
$$

Then there are functions $u_{n} \in H^{1}\left(\mathcal{C}_{n}\right)$ (here $\left.\mathcal{C}_{n}=\mathcal{C}_{\lambda_{n}}\right)$ such that

$$
\int_{\mathcal{C}_{n}}\left|u_{n}\right|^{p} d \mu=1 \text { and } I\left(\lambda_{n}\right) \leq \int_{\mathcal{C}_{n}}\left|\nabla u_{n}\right|^{2}+u_{n}^{2} d \mu \leq S_{p}\left(\mathbb{R}^{N}\right)-\varepsilon_{0} .
$$

Now we need a more detailed concentration-compactness lemma than the one in [21] and along the lines of lemmas 4.1 and 4.2 in [24]. The result in [24] is for the $H^{1}\left(\mathbb{R}^{N}\right)$ setting, but the proof carries over to our situation, too. We omit it here. For $r>0$ and $y_{n, i} \in \mathcal{C}_{n}$, let $\Omega_{n, i}(r)$ be $\psi_{y_{n, i}, r, \lambda_{n}}\left(B_{r}(0)\right)$.

LEMMA 4.2 Let $\lambda_{n} \rightarrow \infty$ and $u_{n} \in H^{1}\left(\mathcal{C}_{n}\right)$ be uniformly bounded (with norm given by $\left.\|u\|^{2}=\int_{\mathcal{C}_{n}}|\nabla u|^{2}+\left|u_{n}\right|^{2} d \mu\right)$. Assume $\int_{\mathcal{C}_{n}}\left|u_{n}\right|^{p} d \mu=1$. Then there is a subsequence (still denoted by $\left(u_{n}\right)$ ), a nonnegative, nonincreasing sequence $\left(\alpha_{i}\right)$ satisfying $\sum_{i=1}^{\infty} \alpha_{i}=1$, and sequences $\left(y_{n, i}\right)_{i} \subset \mathcal{C}_{n}$ associated with each $\alpha_{i}>0$ satisfying

$$
\liminf _{n \rightarrow \infty}\left|y_{n, i}-y_{n, j}\right|=\infty \quad \text { for any } i \neq j
$$

such that the following property holds: If $\alpha_{s}>0$ for some $s \geq 1$, then for any $\varepsilon>0$ there exist $R>0$, for all $r \geq R$ and all $r^{\prime} \geq R$, such that

$$
\begin{array}{r}
\left.\limsup _{n \rightarrow \infty} \sum_{i=1}^{s}\left|\alpha_{i}-\int_{\Omega_{n, i}(r)}\right| u_{n}\right|^{p} d \mu \mid \\
+\left.\left|\left(1-\sum_{i=1}^{s} \alpha_{i}\right)-\int_{\mathcal{C}_{n} \backslash \bigcup_{i=1}^{s} \Omega_{n, i}\left(r^{\prime}\right)}\right| u_{n}\right|^{p+1} d \mu \mid<\varepsilon .
\end{array}
$$

In Lemma 4.2, fix $s>0$ with $\alpha_{s}>0$ such that

$$
\sum_{i=1}^{s} \alpha_{i}>\left(\frac{I_{0}}{S_{p}\left(\mathbb{R}^{N}\right)}\right)^{\frac{p}{2}}
$$

For $\alpha_{s}>\varepsilon>0$, let $R>0$ and $\left(y_{n, i}\right)_{i} \subset \mathcal{C}_{n}$ such that for all $r, r^{\prime}>R$, we have

$$
\begin{aligned}
\left.\lim _{n \rightarrow \infty} \sum_{i=1}^{s}\left|\alpha_{i}-\int_{\Omega_{n, i}(r)}\right| u_{n}\right|^{p} d \mu \mid & \\
+ & \left.\left|\left(1-\sum_{i=1}^{s} \alpha_{i}\right)-\int_{\mathcal{C}_{n} \backslash \bigcup_{i=1}^{s} \Omega_{n, i}\left(r^{\prime}\right)}\right| u_{n}\right|^{p} d \mu \mid<\varepsilon .
\end{aligned}
$$


We now consider a cutoff function $\rho$ on $\mathbb{R}^{N}$ that is identically 1 inside $B_{R}(0)$ and 0 outside $B_{2 R}(0)$ and $|\nabla \rho| \leq \frac{2}{R}$ at any point. For $1 \leq i \leq s$, define $\psi=\psi_{y_{n, i}, 2 R, \lambda_{n}}$ as before, and let $w_{n, i}(x)=\rho(x) u_{n}(\psi(x))$ designate functions with compact support in $\mathbb{R}^{N}$. By a direct computation, we get

$$
\int_{\mathbb{R}^{N}}\left|\nabla w_{n, i}\right|^{2}+w_{n, i}^{2} d x \leq \int_{\Omega_{n, i}(2 R)}\left|\nabla u_{n}\right|^{2}+u_{n}^{2} d \mu+o(1)+\frac{C}{R}
$$

with $C$ independent of $n, \varepsilon$, and $R$, and $o(1) \rightarrow 0$ as $n \rightarrow \infty$. Also,

$$
\int_{\mathbb{R}^{N}}\left|w_{n, i}\right|^{p} d x \geq \int_{\Omega_{n, i}(R)}\left|u_{n}\right|^{p} d \mu+o(1) .
$$

Since

$$
\int_{\mathbb{R}^{N}}\left|\nabla w_{n, i}\right|^{2}+w_{n, i}^{2} d x \geq\left(\int_{\mathbb{R}^{N}}\left|w_{n, i}\right|^{p} d x\right)^{\frac{2}{p}} S_{p}\left(\mathbb{R}^{N}\right)
$$

we obtain

$$
\int_{\Omega_{n, i}(2 R)}\left|\nabla u_{n}\right|^{2}+u_{n}^{2} d \mu+o(1)+\frac{C}{R} \geq
$$

$$
\left(\int_{\Omega_{n, i}(R)}\left|u_{n}\right|^{p} d \mu+o(1)\right)^{\frac{2}{p}} S_{p}\left(\mathbb{R}^{N}\right)
$$

Therefore,

$$
\int_{\mathcal{C}}\left|\nabla u_{n}\right|^{2}+u_{n}^{2} d \mu \geq\left(\sum_{i=1}^{s} \int_{\Omega_{n, i}(R)}\left|u_{n}\right|^{p} d \mu\right)^{\frac{2}{p}} S_{p}\left(\mathbb{R}^{N}\right)+o(1)-\frac{s C}{R} .
$$

From (4.8) we get

$$
\int_{\mathcal{C}}\left|\nabla u_{n}\right|^{2}+u_{n}^{2} d \mu \geq\left(\sum_{i=1}^{s} \alpha_{i}-\varepsilon\right)^{\frac{2}{p}} S_{p}\left(\mathbb{R}^{N}\right)+o(1)-\frac{s C}{R} .
$$

Letting $n \rightarrow \infty$ and then $R \rightarrow \infty$, we obtain

$$
I_{0} \geq\left(\sum_{i=1}^{s} \alpha_{i}-\varepsilon\right)^{\frac{2}{p}} S_{p}\left(\mathbb{R}^{N}\right)
$$

Now, let $\varepsilon \rightarrow 0$ to get

$$
I_{0} \geq\left(\sum_{i=1}^{s} \alpha_{i}\right)^{\frac{2}{p}} S_{p}\left(\mathbb{R}^{N}\right)
$$


which contradicts (4.7).

\section{Symmetry Breaking}

For symmetry breaking, we have Theorem 1.3(i) and (ii). The results of (i) and (ii) will be proved using different ideas. For Theorem 1.3(i), the idea is to use bifurcation techniques and to show that for certain $(a, b)$, by perturbing the radial solution $v_{a}$ given in (2.11), there are directions in which the energy decreases. Since $S(a, b)$ is achieved, the minimizer cannot be radial. This approach has been used for other problems, for example, for bifurcation of positive solutions on annular domains in [20]. On the other hand, for Theorem 1.3(ii), we shall employ an idea in [4] by Brezis and Nirenberg (in which they studied a problem with a nearly critical exponent on annular domains) to compare the radial least energy and $S(a, b)$. A continuity argument then gives the conclusion.

We first give the proof of Theorem 1.3(i). We work in $H^{1}(\mathcal{C})$ here. The linearization of (2.3) at the radial solution $v_{a}$ decomposes by separation of variables into infinitely many ODEs. Denote by $\alpha_{k}=k(N-2+k)$ the $k^{\text {th }}$ eigenvalue of $-\Delta_{\theta}$ on $\mathbb{S}^{N-1}$. For $k \geq 0$, we denote by $\mu_{k}$ and $f_{k}$ the first eigenvalue and the corresponding (positive) eigenfunction in the eigenvalue problem of $\mu$,

$$
-f_{t t}+\lambda^{2} f+\alpha_{k} f-(p-1) v_{a}^{p-2} f=\mu f .
$$

This eigenvalue problem is well defined since $v_{a}(t) \rightarrow 0$ as $|t| \rightarrow \infty$. First, we show that there are $a_{0}$ and a function $a<h(a)<a+1$ defined for $a<a_{0}$ such that $a<a_{0}$ and $a<b<h(a)$ imply $\mu_{1}<0$. Indeed,

$$
\mu_{k}=\inf _{f \in H^{1}(\mathbb{R}) \backslash\{0\}} \frac{\int_{\mathbb{R}} f_{t}^{2}+\lambda^{2} f^{2}+\alpha_{k} f^{2}-(p-1) v_{a}^{p-2} f^{2} d t}{\int_{\mathbb{R}} f^{2} d t} .
$$

We use $v_{a}$ as a test function, and since

$$
\int_{\mathcal{C}} v_{a, t}^{2}+\lambda^{2} v_{a}^{2} d \mu=\int_{\mathcal{C}} v_{a}^{p} d \mu
$$

we obtain

$$
\mu_{k} \leq-(p-2) \frac{\int_{\mathcal{C}} v_{a}^{p} d \mu}{\int_{\mathcal{C}} v_{a}^{2} d \mu}+\alpha_{k} .
$$

Since $\alpha_{0}=0$, clearly we have $\mu_{0}<0$. We also have $\alpha_{1}=N-1$, and by a direct calculation using (2.11), (5.1) gives

$$
\mu_{1} \leq-\frac{N(1+a-b)(N-2-2 a)^{2}}{(N-2(1+a-b))(N-(1+a-b))}+N-1 .
$$

Note that the right-hand side in (5.2) is negative for

$$
a<a_{0}:=\frac{N-2}{2}-\frac{N-1}{2} \sqrt{\frac{N-2}{N}}
$$


and

$$
a \leq b<h(a):=1+a-\frac{2 N}{l(a)+\sqrt{l^{2}(a)-8}},
$$

where

$$
l(a)=\frac{(N-2-2 a)^{2}}{N-1}+3 .
$$

Hence $\mu_{1}$ is negative for $a$ and $b$ in this range. Note also that $a+1-h(a) \rightarrow 0$ as $a \rightarrow-\infty$. The $a_{0}$ and $h(a)$ above will be shown to have the property stated in Theorem 1.3(i).

Define $w_{k}=\phi_{k}(\theta) f_{k}$, where $\phi_{k}$ is an eigenfunction of $-\Delta_{\theta}$ on $\mathbb{S}^{N-1}$ with eigenvalue $\alpha_{k}$. $\left(\phi_{0}(\theta)\right.$ is just a positive constant and $\phi_{1}(\theta)$ is a first harmonic.) We get

$$
-\Delta w_{k}+\lambda^{2} w_{k}-(p-1) v_{a}^{p-2} w_{k}=\mu_{k} w_{k} .
$$

We now have the following:

LEMMA 5.1 For $s$ small, there is $\delta=\delta(s)$ such that $\delta(0)=\delta^{\prime}(0)=0$ and

$$
\int_{\mathcal{C}}\left|v_{a}+\delta(s) w_{0}+s w_{1}\right|^{p} d \mu=1 .
$$

If, in addition, $(a, b)$ is such that $\mu_{1}<0$ (which holds for $a<a_{0}$ and $a \leq b<$ $h(a))$, then for s sufficiently small,

$$
F\left(v_{a}+\delta(s) w_{0}+s w_{1}\right)<F\left(v_{a}\right) .
$$

Proof of Theorem 1.3(i): By the above lemma, for $s$ small $\mid v_{a}+\delta(s) w_{0}+$ $\left.s w_{1}\right|_{L^{p}}=1$. Then (5.6) shows $S(a, b)<R(a, b)$. Since $S(a, b)$ is achieved, the minimizer is nonradial.

Proof OF Lemma 5.1: Set

$$
G(\delta, s)=\int_{\mathcal{C}}\left|v_{a}+\delta w_{0}+s w_{1}\right|^{p} d \mu .
$$

We have $G(0,0)=1$ and $\frac{\partial G}{\partial \delta}(0,0)=p \int_{\mathcal{C}} v_{a}^{p-1} w_{0} d \mu>0$, since $w_{0}>0$. By the implicit function theorem, there is an open $s$-interval around 0 where $\delta=\delta(s)$ is differentiable and

$$
G(\delta(s), s)=1 .
$$

Furthermore, by a direct computation and $\phi_{1}(-\theta)=-\phi_{1}(\theta)$, we have

$$
\frac{\partial G}{\partial s}(0,0)=p \int_{\mathcal{C}} v_{a}^{p-1} w_{1} d \mu=p \int_{\mathcal{C}} v_{a}^{p-1} \phi_{1}(\theta) f_{1} d \mu=0 .
$$

Differentiating (5.7) we get

$$
\frac{\partial G}{\partial \delta}(\delta(s), s) \delta^{\prime}(s)+\frac{\partial G}{\partial s}(\delta(s), s)=0 .
$$


Hence

$$
\frac{\partial G}{\partial \delta}(0,0) \delta^{\prime}(0)+\frac{\partial G}{\partial s}(0,0)=0,
$$

which implies $\delta^{\prime}(0)=0$. To show (5.6) we need $\delta^{\prime \prime}(0)$. Differentiating (5.8) with respect to $s$ again and setting $s=0$, we get

$$
\frac{\partial G}{\partial \delta}(0,0) \delta^{\prime \prime}(0)+\frac{\partial^{2} G}{\partial s^{2}}(0,0)=0 .
$$

We have

$$
\frac{\partial^{2} G}{\partial s^{2}}(0,0)=p(p-1) \int_{\mathcal{C}} u_{a}^{p-2} w_{1}^{2} d \mu \quad \text { and } \quad \frac{\partial G}{\partial \delta}(0,0)=p \int_{\mathcal{C}} u_{a}^{p-1} w_{0} d \mu .
$$

Thus,

$$
\delta^{\prime \prime}(0)=-\frac{p(p-1) \int_{\mathcal{C}} v_{a}^{p-2} w_{1}^{2} d \mu}{p \int_{\mathcal{C}} v_{a}^{p-1} w_{0} d \mu}
$$

Now,

$$
\begin{aligned}
& F\left(v_{a}+\delta(s) w_{0}+s w_{1}\right)= \\
& F\left(v_{a}\right)+s^{2} \int_{\mathcal{C}}\left|\nabla w_{1}\right|^{2}+\lambda^{2} w_{1}^{2} d \mu+2 \delta(s) \int_{\mathcal{C}} \nabla v_{a} \cdot \nabla w_{0}+\lambda^{2} v_{a} w_{0} d \mu \\
& \quad+2 s \int_{\mathcal{C}} \nabla v_{a} \cdot \nabla w_{1}+\lambda^{2} v_{a} w_{1} d \mu+\delta^{2}(s) \int_{\mathcal{C}}\left|\nabla w_{0}\right|^{2}+\lambda^{2} w_{0}^{2} d \mu \\
& +2 s \delta(s) \int_{\mathcal{C}} \nabla w_{0} \cdot \nabla w_{1}+\lambda^{2} w_{0} w_{1} d \mu .
\end{aligned}
$$

Since $v_{a}$ is radial,

$$
\int_{\mathcal{C}} \nabla v_{a} \cdot \nabla w_{1}+\lambda^{2} v_{a} w_{1} d \mu=\int_{\mathcal{C}} v_{a}^{p-1} w_{1} d \mu=0
$$

therefore the fourth term is 0 . Also, the fifth and the sixth terms are higher order.

Hence

$$
\begin{aligned}
& F\left(v_{a}+\delta(s) w_{0}+s w_{1}\right)= \\
& \quad F\left(v_{a}\right)+s^{2} \int_{\mathcal{C}}\left|\nabla w_{1}\right|^{2}+\lambda^{2} w_{1}^{2} d \mu+2 \delta(s) \int_{\mathcal{C}} \nabla v_{a} \nabla w_{0}+\lambda^{2} v_{a} w_{0} d \mu+o\left(s^{2}\right) .
\end{aligned}
$$

From (5.5) we get

$$
\int_{\mathcal{C}}\left|\nabla w_{1}\right|^{2}+\lambda^{2} w_{1}^{2} d \mu=(p-1) \int_{\mathcal{C}} v_{a}^{p-2} w_{1}^{2} d \mu+\mu_{1} \int_{\mathcal{C}} w_{1}^{2} d \mu .
$$


Since $v_{a}$ is a solution of (2.3), we have

$$
\int_{\mathcal{C}} \nabla v_{a} \cdot \nabla w_{0}+\lambda^{2} v_{a} w_{0} d \mu=\int_{\mathcal{C}} v_{a}^{p-1} w_{0} d \mu
$$

Using the equalities above and

$$
\delta(s)=-s^{2} \frac{(p-1) \int_{\mathcal{C}} v_{a}^{p-2} w_{1}^{2} d \mu}{2 \int_{\mathcal{C}} v_{a}^{p-1} w_{0} d \mu}+o\left(s^{2}\right),
$$

we obtain for $s$ sufficiently small

$$
F\left(v_{a}+\delta(s) w_{0}+s w_{1}\right)=F\left(v_{a}\right)+s^{2} \mu_{1} \int_{\mathcal{C}} w_{1}^{2} d \mu+o\left(s^{2}\right)<F\left(v_{a}\right)
$$

The proof of Lemma 5.1 is complete.

Proof of THEOREM 1.3(ii): First we note that by a direct computation using (2.13) we always have for all $a<0$

$$
R(a, a)>S(a, a)=S(0,0) .
$$

We argue that for any $a_{0}<0$, there is $\varepsilon_{0}>0$ such that for all $\left|(a, b)-\left(a_{0}, a_{0}\right)\right|<\varepsilon_{0}$ with $a<b, S(a, b)$ is achieved by a nonradial function. As $(a, b) \rightarrow\left(a_{0}, a_{0}\right)$, we have that $R(a, b) \rightarrow R\left(a_{0}, a_{0}\right)>S\left(a_{0}, a_{0}\right)=S(0,0)$. On the other hand, from Theorem 1.1(i) we have that $S(a, b) \rightarrow S\left(a_{0}, a_{0}\right)$ as $(a, b) \rightarrow\left(a_{0}, a_{0}\right)$. Therefore for any $a_{0}<0$, there is $\varepsilon_{0}>0$ such that $S(a, b)<R(a, b)$ if $\left|(a, b)-\left(a_{0}, a_{0}\right)\right|<$ $\varepsilon_{0}$ with $a \leq b$. By Theorem 1.2(i), $S(a, b)$ is achieved, and due to the strict inequality, the minimizer for $S(a, b)$ is nonradial.

\section{Symmetry of Solutions}

We use the moving plane method [14] to show that for $a \leq b<a+1$ any positive solution of (2.3) on the cylinder $\mathcal{C}$ is symmetric about some $t=$ const, so up to a translation in the $t$-direction, the solution is even in $t$ and satisfies the monotonicity property. Together with the discussion in Section 2, we get that any solution of (1.8) satisfying $u(x)>0$ for $x \in \mathbb{R}^{N} \backslash\{0\}$, up to a dilation (2.4), satisfies the modified inversion symmetry in Theorem 1.4. Our argument follows closely the method in [10] though we have a differential equation defined on a manifold $\mathcal{C}$, while in [10] equations in $\mathbb{R}^{N}$ were treated.

Let $v$ be a positive solution of (2.3). For $\mu<0$ and $x=(t, \theta) \in \mathcal{C}$, denote $x^{\mu}=(2 \mu-t, \theta) \in \mathcal{C}$, the reflection of $x$ relative to the hyperplane $t=\mu$. We let

$$
w_{\mu}(x)=v\left(x^{\mu}\right)-v(x),
$$

a function defined on the region $\Sigma_{\mu}=\{(t, \theta) \in \mathcal{C}: t<\mu\}$. Clearly, $w(x)=0$ for any $x \in T_{\mu}=\partial \Sigma_{\mu}=\{(t, \theta) \in \mathcal{C}: t=\mu\}$. We have the following:

LEMMA 6.1 There is $R_{0}>0$ independent of $\mu$ such that if $w_{\mu}$ has a negative local minimum at $\left(t_{0}, \theta_{0}\right)$, then $\left|t_{0}\right| \leq R_{0}$. 
PROOF: First, by elliptic regularity theory and the fact that

$$
\int_{\tau \leq t \leq \tau+1} v^{2^{*}} d \mu \rightarrow 0 \quad \text { as }|\tau| \rightarrow \infty,
$$

we have $v(t, \theta) \rightarrow 0$ as $|t| \rightarrow \infty$. Then we take $R_{0}$ to be such that

$$
v(t, \theta)<\left(\frac{\lambda^{2}}{p-1}\right)^{\frac{1}{p-2}}
$$

for all $|t| \geq R_{0}$. Since $v$ is a solution of (2.3), $w_{\mu}$ satisfies

$$
-\Delta w_{\mu}+\lambda^{2} w_{\mu}-a(x) w_{\mu}=0
$$

in $\Sigma_{\mu}$, where

$$
a(x)=(p-1) \int_{0}^{1}\left[u(x)+s\left(u\left(x^{\mu}\right)-u(x)\right)\right]^{p-2} d s .
$$

Assume $x_{0}=\left(t_{0}, \theta_{0}\right) \in \Sigma_{\mu}$ is a minimum such that $w_{\mu}\left(x_{0}\right)<0$ and $\left|t_{0}\right|>R_{0}$. Then

$$
v\left(x_{0}^{\mu}\right)<v\left(x_{0}\right)<\left(\frac{\lambda^{2}}{p-1}\right)^{\frac{1}{p-2}} .
$$

Therefore,

$$
a\left(x_{0}\right)<\lambda^{2}
$$

Since $\Delta w_{\mu}\left(x_{0}\right) \geq 0$, we obtain

$$
\lambda^{2} w_{\mu}\left(x_{0}\right)-a\left(x_{0}\right) w_{\mu}\left(x_{0}\right) \geq 0,
$$

which means $\lambda^{2} \leq a\left(x_{0}\right)$, contradicting (6.2).

We shall need the following:

Maximum Principle. If $w_{\mu}$ is nonnegative solution of (6.1) and $w_{\mu}$ is zero at some point in $\Sigma_{\mu}$, then $w_{\mu} \equiv 0$.

Hopf Lemma. If $w_{\mu}$ is positive on $\Sigma_{\mu}$, then $\partial w_{\mu} / \partial t<0$ at any point on $T_{\mu}$.

Proof of Theorem 1.4: Since for $t \rightarrow-\infty$ we have $w_{\mu}(t, \theta) \rightarrow 0$ and $w(x)=0$ for all $x \in T_{\mu}$, Lemma 6.1 implies $w_{\mu}(x) \geq 0$ for $x \in \Sigma_{\mu}$ with all $\mu \leq-R_{0}$. Let $\mu_{0}$ be the largest $\mu$ with the property that $w_{\mu}$ is nonnegative on $\Sigma_{\mu}$. Clearly such $\mu_{0}$ exists since $v(t, \theta) \rightarrow 0$ as $t \rightarrow \infty$. We argue that

$$
\begin{array}{ll}
w_{\mu}(x)>0 & \text { for } x \in \Sigma_{\mu}, \mu<\mu_{0}, \\
w_{\mu_{0}} \equiv 0 & \text { on } \Sigma_{\mu_{0}} .
\end{array}
$$

Since $w_{\mu} \geq 0$ for all $\mu<\mu_{0}$, it follows that $v_{t} \geq 0$ for all $t \leq \mu_{0}$. To prove $(*)$, assume there is $\delta>0$ such that for some $\left(t_{0}, \theta_{0}\right)$, we have $t_{0}<\mu_{0}-\delta$ and 
$w_{\mu_{0}-\delta}\left(t_{0}, \theta_{0}\right)=0$. By the maximum principle it follows that $w_{\mu_{0}-\delta} \equiv 0$. This implies that $v\left(\mu_{0}-2 \delta, \theta_{0}\right)=v\left(\mu_{0}, \theta_{0}\right)$. Since $\partial v / \partial t \geq 0$, it follows that

$$
\frac{\partial v}{\partial t}\left(t, \theta_{0}\right)=0 \quad \text { for all } t \in\left[\mu_{0}-2 \delta, \mu_{0}\right]
$$

Therefore

$$
\frac{\partial w_{\mu_{0}-2 \delta}}{\partial t}\left(\mu_{0}-2 \delta, \theta_{0}\right)=0
$$

By the Hopf lemma we get $w_{\mu_{0}-2 \delta} \equiv 0$. Continuing in this fashion, we obtain that $v$ is independent of $t$, which is not possible. Therefore, $\partial w_{\mu} / \partial t<0$ on $T_{\mu}$ for $\mu<\mu_{0}$ and then $v_{t}>0$ on $\Sigma_{\mu}$.

For $(* *)$, assume $w_{\mu_{0}} \not \equiv 0$. By the maximum principle and the Hopf lemma, $w_{\mu_{0}}>0$ on $\Sigma_{\mu_{0}}$ and $\partial w_{\mu_{0}} / \partial t<0$ on $T_{\mu_{0}}$. From the definition of $\mu_{0}$, there is a sequence $\mu_{k} \searrow \mu_{0}$ and there are points $x_{k} \in \Sigma_{\mu_{k}}$, absolute minima for $w_{\mu_{k}}$, such that $w_{\mu_{k}}\left(x_{k}\right)<0$. By Lemma 6.1 we have that $\left(x_{k}\right)$ is a bounded sequence; hence (by passing to a subsequence) we can assume it converges to some point $x_{0}$. It follows that $x_{0} \in T_{\mu_{0}}$ and $w_{\mu_{0}, t}\left(x_{0}\right)=0$, which is a contradiction.

Eventually after a translation in the $t$-direction, we can assume $\mu_{0}=0$. Therefore $v$ is even in $t$ and monotonically decreasing for $t>0$.

Translations in $t$ on $\mathcal{C}$ correspond to dilations in $\mathbb{R}^{N}$; hence up to a dilation $u(x) \rightarrow \tau^{\frac{N-2-2 a}{2}} u(\tau x)$, positive solutions of (1.8) have the modified inversion symmetry as given in Theorem 1.4.

\section{The Cases $N=1$ and $N=2$}

\subsection{The Case $N=1$}

In one dimension, equation (1.8) becomes

$$
-\left(|x|^{-2 a} u^{\prime}\right)^{\prime}=|x|^{-b p} u^{p-1}, \quad u \geq 0, \quad \text { in } \mathbb{R} .
$$

We have a rather complete answer for the problem. In fact, we can identify all solutions of (7.1). We look for solutions $u$ that are critical points for the energy in $\mathcal{D}_{a}^{1,2}(\mathbb{R})$

$$
E_{a, b}(u)=\frac{\int_{\mathbb{R}}|x|^{-2 a}\left|u^{\prime}\right|^{2} d x}{\left(\int_{\mathbb{R}}|x|^{-b p}|u|^{p} d x\right)^{2 / p}} .
$$

The parameter range is

$$
a<-\frac{1}{2}, \quad a+\frac{1}{2}<b \leq a+1, \quad \text { and } \quad p=\frac{2}{-1+2(b-a)} .
$$

We first observe that $E_{a, b}(u)$ is invariant under the following rather nonstandard dilations: for $\left(\tau_{-}, \tau_{+}\right) \in(0, \infty)^{2}$

$$
u(x) \rightarrow u_{\tau_{-}, \tau_{+}}(x)= \begin{cases}\tau_{-}^{-\frac{1+2 a}{2}} u\left(\tau_{-} x\right), & x<0, \\ \tau_{+}^{-\frac{1+2 a}{2}} u\left(\tau_{+} x\right), & x>0 .\end{cases}
$$


That is, dilations can be made independently for $x<0$ and $x>0$ so that $E_{a, b}(u)$ is still invariant.

Note that for $N=1$ the cylinder $\mathcal{C}=\mathbb{R} \times \mathbb{S}^{0}=\mathbb{R} \cup \mathbb{R}$ is the union of two real lines. We denote the two components by $\mathcal{C}_{-}$and $\mathcal{C}_{+}$corresponding to $\mathbb{R}_{-}$and $\mathbb{R}_{+}$in $\mathbb{R}$, respectively. The coordinates for $\mathcal{C}_{-}$and $\mathcal{C}_{+}$are $y=(t,-1) \in \mathcal{C}_{-}$and $y=(t, 1) \in \mathcal{C}_{+}$. For simplicity, we write them as $t_{1}$ (for $\left.(t,-1)\right)$ and $t_{2}($ for $(t, 1))$. To be more precise, for a function $w(y)$ defined on $\mathcal{C}$ we write $w(y)=w_{1}\left(t_{1}\right)$ when $y=t_{1} \in \mathcal{C}_{-}$and $w(y)=w_{2}\left(t_{2}\right)$ when $y=t_{2} \in \mathcal{C}_{+}$. To a function $u \in \mathcal{D}_{a}^{1,2}(\mathbb{R})$, we associate a function $w$ (corresponding to a pair of $w_{1}, w_{2}$ ) defined on $\mathcal{C}$ by

$$
\begin{array}{ll}
u(x)=(-x)^{(1+2 a) / 2} w_{1}(-\ln (-x)) & \text { for } x<0, \\
u(x)=x^{(1+2 a) / 2} w_{2}(-\ln x) & \text { for } x>0,
\end{array}
$$

and $t_{1}=-\ln |-x|$ for $x<0$ and $t_{2}=-\ln x$ for $x>0$. Then equation (7.1) is equivalent to the system of autonomous equations: for $i=1,2$,

$$
-\frac{d^{2} w_{i}}{d t_{i}^{2}}+\left(\frac{1+2 a}{2}\right)^{2} w_{i}=\left|w_{i}\right|^{p-2} w_{i} .
$$

Critical points of $E_{a, b}(u)$ on $D_{a}^{1,2}(\mathbb{R})$ now correspond to critical points of a new energy functional on $H^{1}(\mathcal{C})$

$$
F_{a, b}(w)=\frac{\int_{\mathcal{C}}|\nabla w|^{2}+\left(\frac{1+2 a}{2}\right)^{2}|w|^{2} d \mu}{\left(\int_{\mathcal{C}}|w|^{p} d \mu\right)^{2 / p}}, \quad w \in H^{1}(\mathcal{C}) .
$$

It is easy to see that both integrals in the numerator and the denominator are decoupled as two integrals for $w_{1}$ and $w_{2}$. Each of the two ODEs of (7.4) has the zero solution, and according to $(2.7)$ with $\lambda=-(1+2 a) / 2$, the only (positive) homoclinic solutions are translates of

$$
\begin{aligned}
v(t)= & \left(\frac{(1+2 a)^{2}}{4(1-2(1+a-b))}\right)^{\frac{1-2(1+a-b)}{4(1+a-b)}} \\
& \left(\cosh \frac{(1+2 a)(1+a-b)}{1-2(1+a-b)} t\right)^{-\frac{1-2(1+a-b)}{2(1+a-b)}} .
\end{aligned}
$$

The minimizers of $F_{a, b}(w)$ are achieved by $w$, for which one of two components $w_{1}$ or $w_{2}$ is identically zero and the other is a translate of $v(t)$ given above. According to (2.9), the infimum is

$$
\begin{aligned}
S(a, b)= & \frac{(-1-2 a)^{2(b-a)}}{2^{2(1+a-b)}(-1+2(b-a))^{-1+2(b-a)}(1+a-b)^{2(1+a-b)}} \\
& \left(\frac{\Gamma^{2}\left(\frac{1}{2(1+a-b)}\right)}{\Gamma\left(\frac{1}{1+a-b}\right)}\right)^{2(1+a-b)}
\end{aligned}
$$


We observe that as $b \searrow a+\frac{1}{2}$, we obtain $S(a, b) \rightarrow-1-2 a$. Note that when both $w_{1}$ and $w_{2}$ are nonzero and are (possibly different) translates of $v(t)$ in (7.5) we get the energy $F_{a, b}(w)$ to be higher

$$
R(a, b)=2^{2(1+a-b)} S(a, b),
$$

which is the least energy in the radial class. On this energy level, there is a twoparameter family of positive solutions according to the two parameters that control by how much $w_{1}$ and $w_{2}$ are translated from (7.5). Correspondingly, $u(x)$ defined in (7.3) is a two-parameter family of solutions for (7.1), which after a dilation given by $(7.2)$ for some $\left(\tau_{-}, \tau_{+}\right) \in(0, \infty)^{2}$ is radial in $\mathbb{R}$.

Summarizing all these, we can state the main results for $N=1$ now.

THEOREM 7.1 (Best Constants and Nonexistence of Extremal Functions)

(i) $S(a, b)$ is continuous in the full parameter domain.

(ii) For $b=a+1$, we have $S(a, a+1)=\left(\frac{1+2 a}{2}\right)^{2}$, and $S(a, a+1)$ is not achieved.

(iii) For $b \rightarrow\left(a+\frac{1}{2}\right)^{+}$, we get $S(a, b) \rightarrow-1-2 a$.

THEOREM 7.2 (Best Constants and Existence of Extremal Functions) For $a+\frac{1}{2}<$ $b<a+1, S(a, b)$ is explicitly given in (7.6), and up to a dilation of the form (7.2) it is achieved at a function of the form (7.3) with either $w_{1}=0$ and $w_{2}$ given by (7.5), or vice versa. Consequently, the minimizer for $S(a, b)$ is never radial.

THEOREM 7.3 (Bound State Solutions and Symmetry) Up to a dilation (7.2), the only solution of (7.1) besides the ground state solutions is of the form of (7.3) with both $w_{1}$ and $w_{2}$ given by (7.5). Consequently, all bound state solutions of (7.1), possibly after a dilation given in (7.2), satisfy the modified inversion symmetry.

REMARK 7.4 Due to the degeneracy, the ground state solutions are discontinuous at 0 and identically zero in either $\mathbb{R}_{-}$or $\mathbb{R}_{+}$.

\subsection{The Case $N=2$}

In this case the parameter range is

$$
-\infty<a<0, \quad a<b \leq a+1, \quad \text { and } \quad p=\frac{2}{b-a} .
$$

With no changes in the proofs for the case $N \geq 3$, we have the following results. THEOREM 7.5 (Best Constants and Nonexistence of Extremal Functions)

(i) $S(a, b)$ is continuous in the full parameter domain.

(ii) For $b=a+1$, we have $S(a, a+1)=a^{2}$, and $S(a, a+1)$ is not achieved. THEOREM 7.6 (Best Constants and Existence of Extremal Functions)

(i) For $a<b<a+1, S(a, b)$ is always achieved.

(ii) For $b-a \in(0,1)$ fixed, as $a \rightarrow-\infty, S(a, b)$ is strictly increasing, and

$$
S(a, b)=(-a)^{2(b-a)}\left[S_{p}\left(\mathbb{R}^{2}\right)+o(1)\right] .
$$

One notes in (5.3) that for $N=2$ we have $a_{0}=0$. Therefore we also have the following: 
THEOREM 7.7 (Symmetry Breaking) There is a function $h(a)$ defined for $a<0$, satisfying $a<h(a)<a+1$ for $a<0$ and $a+1-h(a) \rightarrow 0$ as $-a \rightarrow \infty$, such that for any $(a, b)$ satisfying $a<0$ and $a<b<h(a)$, the minimizer for $S(a, b)$ is nonradial.

THEOREM 7.8 (Symmetry Property) For $a<b<a+1$, the minimizer of $S(a, b)$, possibly after a dilation $u(x) \rightarrow \tau^{-a} u(\tau x)$, satisfies the modified inversion symmetry:

$$
u\left(\frac{x}{|x|^{2}}\right)=|x|^{-2 a} u(x) .
$$

\section{A Related Variational Problem}

In this section we shall consider a related problem that can be solved by using our method and the results we obtained in the previous sections. For $0 \leq a<$ $(N-2) / 2$, special cases of the following problem were considered in [22] and [25]:

For $N \geq 3$, we consider the following problem:

$$
-\operatorname{div}\left(|x|^{-2 a} \nabla w\right)+\gamma|x|^{-2(1+a)} w=|x|^{-b p} w^{p-1}, \quad u \geq 0, \quad \text { in } \mathbb{R}^{N},
$$

where

$$
\begin{gathered}
a<\frac{N-2}{2}, \quad a \leq b<a+1, \quad \gamma>-\left(\frac{N-2-2 a}{2}\right)^{2}, \\
p=\frac{2 N}{N-2+2(b-a)} .
\end{gathered}
$$

The solutions in $\mathscr{D}_{a}^{1,2}\left(\mathbb{R}^{N}\right)$ of this problem are critical points of

$$
E_{a, b, \gamma}(u)=\frac{\int_{\mathbb{R}^{N}}|x|^{-2 a}|\nabla u|^{2}+\gamma|x|^{-2(1+a)} u^{2} d x}{\left(\int_{\mathbb{R}^{N}}|x|^{-b p}|u|^{p} d x\right)^{2 / p}} .
$$

PROPOSITION 8.1 The solutions in $\mathscr{D}_{a}^{1,2}\left(\mathbb{R}^{N}\right)$ of $(8.1)$ are in one-to-one correspondence to solutions in $\mathscr{D}_{\bar{a}}^{1,2}\left(\mathbb{R}^{N}\right)$ of

$$
-\operatorname{div}\left(|x|^{-2 \bar{a}} \nabla u\right)=|x|^{-\bar{b} p} u^{p-1}, \quad u \geq 0, \quad \text { in } \mathbb{R}^{N},
$$

where

$$
\bar{a}=a+\lambda-\sqrt{\lambda^{2}+\gamma}, \quad \bar{b}=b+\lambda-\sqrt{\lambda^{2}+\gamma}, \quad \lambda=\frac{N-2-2 a}{2} .
$$

This correspondence is given by

$$
u(x)=|x|^{\lambda-\sqrt{\lambda^{2}+\gamma}} w(x) .
$$

Direct computations verify the proof, which we omit here.

Due to this proposition, we can put equation (8.1) in the same frame of work as in (1.8), and we can translate all of our results for (1.8) to get corresponding results for (8.1). We note that even in the $a$-nonnegative region, for $\gamma$ sufficiently large 
the minimizer of $E_{a, b, \gamma}(u)$ is nonradial. All of our main theorems are adapted in the obvious way. We leave the statements of these results to the reader.

REMARK 8.2 Proposition 8.1 also holds for $N=1$ and $N=2$ with $a$ and $b$ in the corresponding regions.

REMARK 8.3 For $0 \leq a<\frac{N-2}{2}$, special cases of (8.1) were considered in [22] $(a=b=0$ and $-S(0,1)<\gamma<0)$ and [25] $(a \leq b<a+1$ and $-S(a, a+1)<$ $\gamma<0, a<b<a+1$ with $\gamma>0$, and $0<a=b$ with $0<\gamma \ll 1$ ), but only compactness of minimizing sequences was given.

\section{Final Remarks and Questions}

We finish the paper with some remarks and related open questions.

First, we have given the best constants on the boundary of the $a$-negative region. In view of Theorem 1.3, it seems that there are no closed form minimizers. An interesting question here is, what are the best constants in the interior of the $a$ negative region?

Another question is, in view of Theorem 1.3, what are the optimal parameter values at which the symmetry breaking exactly occurs, namely, the optimal form of $h(a)$ ?

Our analysis indicates that the radial solutions get more and more unstable as $a \rightarrow-\infty$, and this suggests there should be more and more nonradial solutions. We have studied this in [9]. Some of the results in this paper as well as those of [9] have been announced in [8].

Finally, an interesting question is related to the cases $N=1$ and $N=2$. With regard to the Caffarelli-Kohn-Nirenberg inequalities, what are the optimal spaces for $N=1$ with $b=a+\frac{1}{2}$ and for $N=2$ with $b=a$ ?

After this paper was submitted, M. Willem kindly informed us of his preprint [27] and another reference [16] that contain related results to our Theorem 1.1(iii) and 1.2(ii) by using different methods.

Acknowledgment. The authors would like to thank L. Nirenberg for his encouragement and pointing out some references in the preparation of the paper.

\section{Bibliography}

[1] Aubin, T. Problèmes isopérimétriques de Sobolev. J. Differential Geometry 11 (1976), no. 4, 573-598.

[2] Berestycki, H.; Esteban, M. Existence and bifurcation of solutions for an elliptic degenerate problem. J. Differential Equations 134 (1997), no. 1, 1-25.

[3] Brezis, H.; Lieb, E. H. A relation between pointwise convergence of functions and convergence of functionals. Proc. Amer. Math. Soc. 88 (1983), no. 3, 486-490.

[4] Brezis, H.; Nirenberg, L. Positive solutions of nonlinear elliptic equations involving critical exponents. Comm. Pure Appl. Math. 36 (1983), no. 4, 437-477.

[5] Caffarelli, L. A.; Gidas, B.; Spruck, J. Asymptotic symmetry and local behavior of semilinear elliptic equations with critical Sobolev growth. Comm. Pure Appl. Math. 42 (1989), no. 3, 271297. 
[6] Caffarelli, L. A.; Kohn, R.; Nirenberg, L. First order interpolation inequalities with weights. Compositio Math. 53 (1984), no. 3, 259-275.

[7] Caldiroli, P.; Musina, R. On the existence of extremal functions for a weighted Sobolev embedding with critical exponent. Cal. Var. Partial Differential Equations 8 (1999), no. 4, 365-387.

[8] Catrina, F.; Wang, Z.-Q. On the Caffarelli-Kohn-Nirenberg inequalities. C. R. Acad. Sci. Paris Sér. I Math. 330 (2000), no. 6, 437-442.

[9] Catrina, F.; Wang, Z.-Q. Positive bound states having prescribed symmetry for a class of nonlinear elliptic equations in $R^{N}$. Ann. Inst. H. Poincaré Anal. Non Linéaire, in press.

[10] Chen, W.; Li, C. Classification of solutions of some nonlinear elliptic equations. Duke Math. J. 63 (1991), no. 3, 615-622.

[11] Chou, K. S.; Chu, C. W. On the best constant for a weighted Sobolev-Hardy inequality. J. London Math. Soc. (2) 48 (1993), no. 1, 137-151.

[12] Dautray, R.; Lions, J.-L. Mathematical analysis and numerical methods for science and technology. Vol. 1. Physical origins and classical methods. Spinger, Berlin, 1985.

[13] Davies, E. B. A review of Hardy inequalities. Preprint.

[14] Gidas, B.; Ni, W. M.; Nirenberg, L. Symmetry of positive solutions of nonlinear elliptic equations in $\mathbb{R}^{N}$. Mathematical analysis and applications, Part A, 369-402. Advances in Mathematics Supplemetary Studies, 7a. Academic Press, New York-London, 1981.

[15] Hardy, G. H.; Littlewood, J. E.; Pólya, G. Inequalities. Second edition. Cambridge, University Press, 1952.

[16] Horiuchi, T. Best constant in weighted Sobolev inequality with weights being powers of distance from the origin. J. Inequal. Appl. 1 (1997), no. 3, 275-292.

[17] Korevaar, N.; Mazzeo, R.; Pacard, F.; Schoen, R. Refined asymptotics for constant scalar curvature metrics with isolated singularities. Invent. Math. 135 (1999), no. 2, 233-272.

[18] Lieb, E. H. Sharp constants in the Hardy-Littlewood-Sobolev and related inequalities. Ann. of Math. (2) 118 (1983), no. 2, 349-374.

[19] Lin, C. S. Interpolation inequalities with weights. Comm. Partial Differential Equations 11 (1986), no. 14, 1515-1538.

[20] Lin, S.-S. Existence of positive nonradial solutions for nonlinear elliptic equations in annular domains. Trans. Amer. Math. Soc. 332 (1992), no. 2, 775-791.

[21] Lions, P.-L. The concentration-compactness principle in the calculus of variations, The locally compact case, Part 1 and Part 2. Ann. Inst. H. Poincaré Anal. Non Linéaire. 1 (1984), 109-145, 223-283.

[22] Lions, P.-L. Concentration compactness principle in the calculus of variations. The limit case. I, II. Rev. Mat. Iberoamericana 1 (1985), no. 1, 145-201; 1 (1985), no. 2, 45-121.

[23] Talenti, G. Best constant in Sobolev inequality. Ann. Mat. Pura Appl. (4) 110 (1976), 353-372.

[24] Wang, Z.-Q. Existence and symmetry of multi-bump solutions for nonlinear Schrödinger equations. J. Differential Equations 159 (1999), no. 1, 102-137.

[25] Wang, Z.-Q.; Willem, M. Singular minimization problems. J. Differential Equations 161 (2000), no. 2, 307-320.

[26] Willem, M. Minimax theorems. Progress in Nonlinear Differential Equations and Their Applications, 24. Birkhäuser, Boston, 1996.

[27] Willem, M. A decomposition lemma and critical minimization problems. Preprint. 
FLORIN CATRINA

Utah State University

Department of Mathematics and Statistics

3900 Old Main Hill

Logan, Utah 84322-3900

E-mail: s 19qg@math . usu . edu

Received February 2000.
ZHI-QIANG WANG

Utah State University

Department of Mathematics and Statistics

3900 Old Main Hill

Logan, Utah 84322-3900

E-mail: wang@math.usu .edu 